\title{
Flexible Generalized Spatial Modulation for Visible Light Communications
}

\author{
Mohamed Al-Nahhal, Student Member, IEEE, Ertugrul Basar, Senior Member, IEEE, \\ and Murat Uysal, Fellow Member, IEEE
}

\begin{abstract}
Adaptive spatial modulation (ASM) varies the modulation size across the transmit light-emitting diodes (LEDs) to improve the overall spectral efficiency of visible light communication (VLC) systems. This paper proposes a novel ASM scheme, referred to as flexible generalized spatial modulation (FGSM), for VLC systems. The proposed FGSM system changes the modulation sizes over the LEDs and the number of active LEDs, to improve the average symbol error rate (SER) and spectral efficiency compared to ASM with a fixed overall number of LEDs. The modulation sizes are selected in order to optimize the average SER under a predefined spectral efficiency value. A closed-form expression of approximate SER is derived along with decoding complexity calculations for the proposed system. Numerical results are provided to confirm the superiority of the proposed system and to support theoretical derivations.
\end{abstract}

Index Terms - Visible light communication, generalized spatial modulation, decoding complexity, adaptive modulation sizes.

\section{INTRODUCTION}

V ISIBLE light communication (VLC) is an emerging wireless access technology considered as a complementary or alternative solution to radio-frequency counterparts [1]. VLC systems use light-emitting diodes (LEDs) as wireless transmitters, while photodetectors (PDs) are employed as receivers. VLC systems are based on intensity modulation and direct detection due to the nature of the LED transmitters and typically employ unipolar signaling, such as on-off keying and other pulse modulation techniques [2].

In indoor environments, such as conference halls, libraries, airports and other similar public places, a large number of ceiling LEDs are typically installed to offer sufficient illumination. This makes multiple-input multiple-output (MIMO) systems a natural candidate for VLC. In [3], Fath and Haas have presented a performance comparison of different MIMO techniques, namely repetition coding (RC), spatial multiplexing (SMUX) and spatial modulation (SM) for VLC systems. In RC, the same signal is transmitted simultaneously from all LEDs, while independent signals are transmitted simultaneously from all LEDs in the SMUX. In the SM technique, a single LED is activated and the index of the activated LED carries information in addition to modulated signals [4, Ch. 6]. SM achieves a better error rate performance in comparison to

Mohamed Al-Nahhal and Murat Uysal are with the Department of Electrical and Electronics Engineering, Ozyegin University, Istanbul 34794, Turkey (emails: mohamed.al-nahhal@ozu.edu.tr; murat.uysal@ozyegin.edu.tr).

Ertugrul Basar is with the CoreLab, Department of Electrical and Electronics Engineering, Koç University, Istanbul 34450, Turkey (e-mail: ebasar@ku.edu.tr).

This work was supported by the Turkish Scientific and Research Council under Grant $215 \mathrm{E} 311$.
SMUX while it yields a higher spectral efficiency with respect to RC.

Recently, an LED grouping SM scheme has been investigated in [5] to decrease the channel correlation effect and enhance the symbol error rate (SER) performance compared to conventional SM. In [6], physical layer security has been investigated for optical SM. Channel-adapted SM for massive MIMO VLC systems has been introduced in [7]. In [8], generalized SM (GSM) has been introduced, where a combination of LEDs is simultaneously activated for transmission. In [9], an optimal collaborative constellation design of GSM has been proposed in order to improve power efficiency for VLC systems. In addition, an ordered-block maximum likelihood (ML) detection has been investigated in order to decrease the detection complexity. It should be noted that the modulation size is fixed in [5]-[9] across all active LEDs.

An adaptive SM (ASM) scheme has been proposed in [10], where the transmitted symbols through the LEDs are modulated using different modulation sizes to improve the spectral efficiency. The ASM scheme activates only a single LED at any given time and transmits the modulated symbol from the activated LED. The size of the used modulation depends on which LED is activated in the ASM scheme. Thus, the ASM scheme exploits the index of a single active LED to carry additional information with respect to the modulated symbol transmitted from that active LED. However, the ASM scheme has a limited spectral efficiency and requires an increased number of transmit LEDs to achieve satisfactory data rates.

In this paper, we propose the so-called flexible generalized spatial modulation (FGSM) for VLC systems to overcome the main limitation of ASM. The proposed FGSM achieves better average SER and higher spectral efficiency in comparison to ASM for the same overall number of LEDs. The proposed system varies the number of active LEDs as well as modulation sizes over the active LEDs. The selection of the modulation sizes is made based on the solution of SER minimization under a predefined value of the spectral efficiency.

The rest of the paper is organized as follows: In Section II, we present the system model for the proposed FGSM. In Section III, we derive the closed-form expression of the approximate average SER and formulate the SER optimization for the selection of modulation size. In addition, we present the decoding complexity analysis for the proposed FGSM. In Section IV, we present numerical results to confirm the accuracy of our derivations and to demonstrate the superiority of FGSM over existing schemes. Finally, we provide concluding remarks in Section V. 


\section{Proposed FGSM System Model}

We consider a VLC system with $L$ LEDs at the transmitter and $P$ PDs at the receiver. The proposed FGSM system basically activates more than a single LED (i.e., activates a combination of LEDs) and transmits symbols selected from variable modulation sizes across LED combinations. The number of active LEDs at any given time is denoted by $L_{a}$, where $1<L_{a}<L$. The proposed system has total of $2^{\eta_{s}}$ LED combinations where $\eta_{s}=\left\lfloor\log _{2}\left(\begin{array}{c}L \\ L_{a}\end{array}\right)\right\rfloor$ is the number of bits transmitted in the spatial domain (i.e., spatial spectral efficiency) and $\lfloor\cdot\rfloor$ denotes the floor operation.

The proposed system employs unipolar pulse amplitude modulation (PAM) and the LED combinations are grouped into $U, 1 \leq U \leq L$, combination groups, each of which uses a different modulation size of unipolar PAM. Assume that the available modulation sizes of the unipolar PAM is $\mathbf{m}=[M(1) \ldots M(U)]$, and the set of constellation symbols that can be transmitted from the $u$-th combination group denoted by $X(u)$ and is given as

$$
X(u) \in\left\{x_{1}^{u}, \ldots, x_{M(u)}^{u}\right\},
$$

where $x_{m}^{u}$ (with the average electrical symbol energy $E_{s}$ ) is the $m$-th symbol transmitted from the $u$-th combination group for $m \in\{1, \ldots, M(u)\}$, and $M(u)$ represents the selected modulation size of the $u$-th combination group. It should be noted that the constellation symbol $x_{m}^{u}=m$ is considered.

Let $g$ represent the LED combination and $\mathcal{I}_{g}$ stand for the set of indices for the active LED combination. Thus, the received signal, $\mathbf{y} \in \mathbb{R}^{P \times 1}$, can be expressed as

$$
\mathbf{y}=\sum_{\mathcal{I}_{g}} \mathbf{h}_{\mathcal{I}_{g}} x_{m}^{u}+\mathbf{w}
$$

where $\mathbf{w} \in \mathbb{R}^{P \times 1}$ is the vector of additive white Gaussian noise samples with zero-mean and variance $\sigma_{w}^{2}$. Here, $\sum_{\mathcal{I}_{g}} \mathbf{h}_{\mathcal{I}_{g}}$ is the summation of $L_{a}$ columns of the VLC channel matrix, $\mathbf{H} \in \mathbb{R}^{P \times L}$, based on the indices of $\mathcal{I}_{g}$.

At the receiver side, the task of the receiver is to find $g$ (which accordingly indicates the indices of $\mathcal{I}_{g}$ and the combination group of $u$ ) and the transmitted symbol $x_{m}^{u}$. Thus, the ML decoder can be formulated as

$$
\{\hat{g}, \hat{m}\}=\arg \underset{\substack{g=1, \ldots, 2^{\eta_{s}} \\ m=1, \ldots, M(u)}}{ }\left\|\mathbf{y}-\sum_{\mathcal{I}_{g}} \mathbf{h}_{\mathcal{I}_{g}} x_{m}^{u}\right\|^{2} .
$$

The number of LED combinations in each combination group $u$ is denoted by $N_{u}$, where $\sum_{u=1}^{U} N_{u}=2^{\eta_{s}}$. Therefore, the overall spectral efficiency, $\eta$ in bits per channel use (bpcu), of the proposed FGSM system can be calculated as

$$
\eta=\left\lfloor\log _{2}\left(\begin{array}{c}
L \\
L_{a}
\end{array}\right)\right\rfloor+\frac{1}{2^{\eta_{s}}} \sum_{u=1}^{U} N_{u} \log _{2} M(u) .
$$

As an example, consider an FGSM system with $L=8$, $L_{a}=2\left(\eta_{s}=4 \mathrm{bpcu}\right), N_{1}=N_{2}=8$ (i.e., $\left.U=2\right)$ and the available modulation sizes of unipolar PAM $\mathbf{m}=[432]$. Here, $N_{1}$ and $N_{2}$ represent the number of LED combinations in the first and second combination groups, respectively. Fig. 1

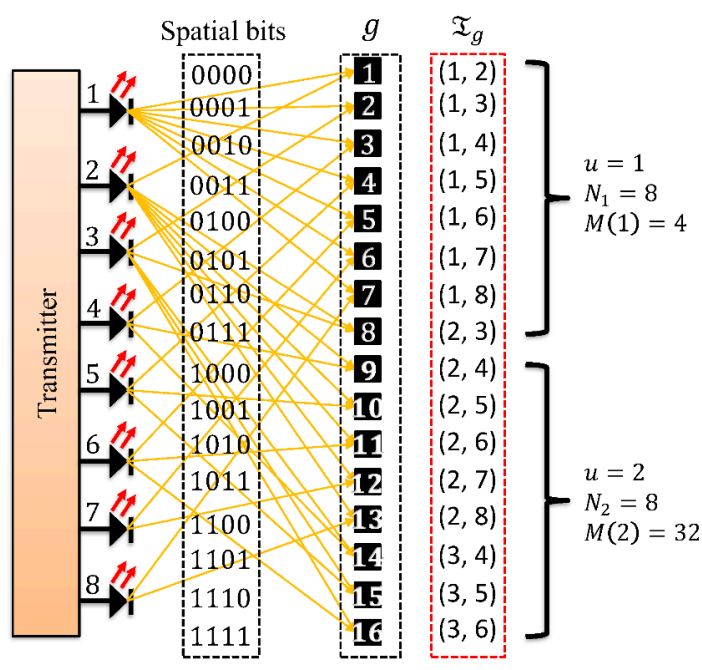

Fig. 1. Proposed FGSM system for $L=8, L_{a}=2, U=2$ and $\mathbf{m}=\left[\begin{array}{ll}4 & 32\end{array}\right]$.

presents the concept of varying the available modulation sizes over the combination groups based on the values of $N_{1}$ and $N_{2}$. It should be noted that the FGSM system requires only $2^{\eta_{s}}$ LED combinations out of $\left(\begin{array}{c}L \\ L_{a}\end{array}\right)$ possibilities. For instance, in Fig. 1, the proposed system with $L=8$ and $L_{a}=2$ requires only 16 LED combinations out of 28 possibilities.

\section{FGSM SYSTEM ANALYSIS}

In this section, we present the derivation of the approximate average SER for the proposed FGSM system. Furthermore, we present the decoding complexity of the proposed FGSM and compare it with that of the ASM.

\section{A. SER Performance}

In the FGSM system, the approximate SER for the $g$-th LED combination, $\mathrm{SER}^{g}$, can be expressed as

$$
\mathrm{SER}^{g} \approx P\left(x_{m}^{g}\right)+P\left(\mathcal{I}_{g}\right)-P\left(x_{m}^{g}\right) P\left(\mathcal{I}_{g}\right), g=1, \ldots, 2^{\eta_{s}},(5)
$$

where $P\left(x_{m}^{g}\right)$ represents the error probability of the symbol $x_{m}^{g}$ and $P\left(\mathcal{I}_{g}\right)$ represents the error probability in $\mathcal{I}_{g}$. Here, $x_{m}^{g}$ is the $m$-th symbol transmitted from the $g$-th LED combination and the symbol $x_{m}^{g}$ is considered as $x_{m}^{g}=m$, $m \in\{1, \ldots, M(g)\}$.

The error probability of the symbol $x_{m}^{g}$ is given by [11, Eq. (4.3-3)]

$$
P\left(x_{m}^{g}\right)=Q\left(\frac{d_{\min }^{g}}{\sqrt{4 \sigma_{w}^{2}}}\right),
$$

where $d_{\min }^{g}$ is the minimum Euclidean distance between any two adjacent symbols. Based on the unipolar PAM constellation where $x_{m}^{g}=m, m \in\{1, \ldots, M(g)\}$ (i.e., $\left|x_{1}^{g}-x_{2}^{g}\right|=1$ ) and under the assumption of correct index detection, $d_{\min }^{g}$ can be expressed as

$$
d_{\min }^{g}=\frac{1}{\sqrt{L_{a}}}\left\|\sum_{\mathcal{I}_{g}} \mathbf{h}_{\mathcal{I}_{g}}\right\| .
$$

By substituting (7) into (6), the error probability of the symbol $x_{m}^{g}$ in (6) becomes 


$$
P\left(x_{m}^{g}\right)=Q\left(\frac{\left\|\sum_{\mathcal{I}_{g}} \mathbf{h}_{\mathcal{I}_{g}}\right\|}{\sqrt{4 L_{a} \sigma_{w}^{2}}}\right) .
$$

The error probability in $\mathcal{I}_{g}$ for the $m$-th symbol can be represented as $Q\left(D_{m}^{g} / \sqrt{4 \sigma_{w}^{2}}\right)$, where $D_{m}^{g}$ is the minimum Euclidean distance between $x_{m}^{g}$ and all possible symbols can be transmitted from the other LED combinations. Thus, for a given combination $g, D_{m}^{g}$ can be expressed as

$$
D_{m}^{g}=\min _{\substack{i=1, \ldots, 2^{\eta_{s}} \\ q=1, \ldots, M(i) \\ i \neq g}} \frac{1}{\sqrt{L_{a}}}\left\|\sum_{\mathcal{I}_{g}} \mathbf{h}_{\mathcal{I}_{g}} x_{m}^{g}-\sum_{\mathcal{I}_{i}} \mathbf{h}_{\mathcal{I}_{i}} x_{q}^{i}\right\| .
$$

The total error probability in $\mathcal{I}_{g}$ becomes

$$
P\left(\mathcal{I}_{g}\right)=\frac{1}{M(g)} \sum_{m=1}^{M(g)} Q\left(\frac{D_{m}^{g}}{\sqrt{4 \sigma_{w}^{2}}}\right) .
$$

Using (8) and (10) into (5), the closed-form expression of the approximate average SER for FGSM can be expressed as

$$
\begin{aligned}
\mathrm{SER} \approx & \sum_{g=1}^{2^{\eta_{s}}} \frac{1}{2^{\eta_{s}}}\left[Q\left(\frac{\left\|\sum_{\mathcal{I}_{g}} \mathbf{h}_{\mathcal{I}_{g}}\right\|}{\sqrt{4 L_{a} \sigma_{w}^{2}}}\right)+\frac{1}{M(g)} \sum_{m=1}^{M(g)} Q\left(\frac{D_{m}^{g}}{\sqrt{4 \sigma_{w}^{2}}}\right)\right. \\
& \left.-\frac{1}{M(g)} Q\left(\frac{\left\|\sum_{\mathcal{I}_{g}} \mathbf{h}_{\mathcal{I}_{g}}\right\|}{\sqrt{4 L_{a} \sigma_{w}^{2}}}\right) \sum_{m=1}^{M(g)} Q\left(\frac{D_{m}^{g}}{\sqrt{4 \sigma_{w}^{2}}}\right)\right] .
\end{aligned}
$$

In the FGSM system, the electrical received signal-to-noise ration (SNR) is given by

$$
\gamma=\frac{E_{s}}{L \sigma_{w}^{2}} \sum_{p=1}^{P}\left|\sum_{l=1}^{L} h_{l, p}\right|^{2}
$$

where $h_{l, p}$ represents the entry of the VLC channel matrix $\mathbf{H}$ between $l$-th LED and $p$-th PD, $l=1, \ldots, L, p=1, \ldots, P$.

\section{B. SER Optimization}

The average SER in (11) can be optimized by selecting the appropriate modulation sizes $\mathbf{m}=[M(1) \ldots M(U)]$ given a predefined value of a spectral efficiency $\eta_{0}$. The optimization problem of the average SER can be formulated as

$$
\mathbf{m}=\arg \min _{\mathbf{m}=[M(1) \ldots M(U)]}\left\{\mathrm{SER} \mid \eta_{0}\right\} .
$$

The optimization problem in (13) is a global one and the objective function is non-convex. Thus, this optimization can be solved by the exhaustive search algorithm [13, Ch. 1]. In our case, the total number of possible sets (i.e., possible sets of the modulation sizes at a given spectral efficiency) is typically low so that exhaustive search can be efficiently employed. If required, for further complexity reduction, we can utilize the concept of variance reduction technique $[14, \mathrm{Ch} .6]$ as detailed below.
Assume that $\mathbf{m}_{k}=\left[M_{k}(1) \ldots M_{k}(U)\right]$ defines the $k$-th possible set of the modulation sizes at a predefined value of the spectral efficiency $\eta_{0}$, where $k=1, \ldots, K$ and $K$ is the total possible sets.

Step1: Define the value of the spectral efficiency $\eta_{0}$ and all possible sets of the modulation sizes $\mathbf{m}_{k}$ for $\forall k=1, \ldots, K$ at $\eta_{0}$.

Step 2: Calculate variance estimate $\mathrm{V}\left(\mathbf{m}_{k}\right)$ of the $k$-th possible set of the modulation size as

$$
\mathrm{V}\left(\mathbf{m}_{k}\right)=\frac{1}{2^{\eta_{s}}} \sum_{u=1}^{U} N_{u}\left(M_{k}(u)-\bar{m}_{k}\right)^{2},
$$

where $\bar{m}_{k}$ represents the average of all elements in $\mathbf{m}_{k}$.

Step 3: Choose $\grave{K}$ sets out of $K$ possible sets of the modulation sizes which have the lowest $\mathrm{V}\left(\mathbf{m}_{k}\right)$ values.

Step 4: Use the chosen $\grave{K}$ sets for (13).

As an example, consider an FGSM system with $L=8$, $U=2\left(N_{1}=2^{\eta_{s}} / 2, N_{2}=2^{\eta_{s}} / 2\right)$, and $\eta=7.5 \mathrm{bpcu}$. There are only 6 possible sets of modulation sizes (i.e., $K=6$ ). Specifically, for $L_{a}=2$, the possible sets are $\mathbf{m}_{1}=\left[\begin{array}{ll}2 & 64\end{array}\right]$, $\mathbf{m}_{2}=\left[\begin{array}{ll}4 & 32\end{array}\right]$ and $\mathbf{m}_{3}=\left[\begin{array}{ll}8 & 16\end{array}\right]$; for $L_{a}=3$, the possible sets are $\mathbf{m}_{4}=\left[\begin{array}{ll}2 & 16\end{array}\right]$ and $\mathbf{m}_{5}=\left[\begin{array}{ll}4 & 8\end{array}\right]$; for $L_{a}=4$, the possible set is $\mathbf{m}_{6}=\left[\begin{array}{ll}2 & 4\end{array}\right]$. If the variance reduction technique is applied, we have $\mathrm{V}\left(\mathbf{m}_{1}\right)=961, \mathrm{~V}\left(\mathbf{m}_{2}\right)=196, \mathrm{~V}\left(\mathbf{m}_{3}\right)=16$, $\mathrm{V}\left(\mathbf{m}_{4}\right)=49, \mathrm{~V}\left(\mathbf{m}_{5}\right)=4$, and $\mathrm{V}\left(\mathbf{m}_{6}\right)=1$. If $K=2$, the chosen possible sets are $\mathbf{m}_{5}=\left[\begin{array}{ll}4 & 8\end{array}\right]$ and $\mathbf{m}_{6}=\left[\begin{array}{ll}2 & 4\end{array}\right]$. Therefore, the search set of 6 possible solutions are now reduced to 2 . As another example, we consider $16 \times 16$ FGSM. For this system, the possible sets of modulation sizes are 11. Specifically, for $L_{a}=2$, the possible sets are $\mathbf{m}_{1}=\left[\begin{array}{ll}32 & 1024\end{array}\right]$, $\mathbf{m}_{2}=\left[\begin{array}{ll}64 & 512\end{array}\right]$ and $\mathbf{m}_{3}=\left[\begin{array}{ll}128 & 256\end{array}\right]$; for $L_{a}=3$, the possible sets are $\mathbf{m}_{4}=\left[\begin{array}{ll}2 & 256\end{array}\right], \mathbf{m}_{5}=\left[\begin{array}{ll}4 & 128\end{array}\right], \mathbf{m}_{6}=\left[\begin{array}{ll}8 & 64\end{array}\right]$ and $\mathbf{m}_{7}=\left[\begin{array}{ll}16 & 32\end{array}\right]$; for $L_{a}=4$, the possible sets are $\mathbf{m}_{8}=\left[\begin{array}{ll}2 & 64\end{array}\right]$, $\mathbf{m}_{9}=\left[\begin{array}{ll}4 & 32\end{array}\right]$ and $\mathbf{m}_{10}=\left[\begin{array}{ll}8 & 16\end{array}\right]$; for $L_{a}=5$, the possible set is $\mathbf{m}_{11}=\left[\begin{array}{ll}2 & 4\end{array}\right]$. If the variance reduction technique is applied, the search set can be reduced to 2 .

\section{Decoding Complexity}

The decoding complexity can be assessed in terms of the number of additions and multiplications required to decode the transmitted signal at the receiver side [15].

From (3), the total number of multiplications and additions of the proposed FGSM system are respectively given as

$$
\begin{gathered}
\operatorname{Mul}_{\mathrm{FGSM}}=2 P \sum_{u=1}^{U} N_{u} M(u), \\
\operatorname{Add}_{\mathrm{FGSM}}=(2 P-1) \sum_{u=1}^{U} N_{u} M(u)+2^{\eta_{s}} P\left(L_{a}-1\right) .
\end{gathered}
$$

Note that the extra complexity required for the FGSM over the ASM is $2^{\eta_{s}} P\left(L_{a}-1\right)$ additions only (no extra multiplications are required); these extra additions come from activating $L_{a}$ LEDs. Thus, the total number of multiplications and additions for the ASM are respectively calculated as

$$
\operatorname{Mul}_{\text {ASM }}=2 P \sum_{u=1}^{U} N_{u} M(u),
$$




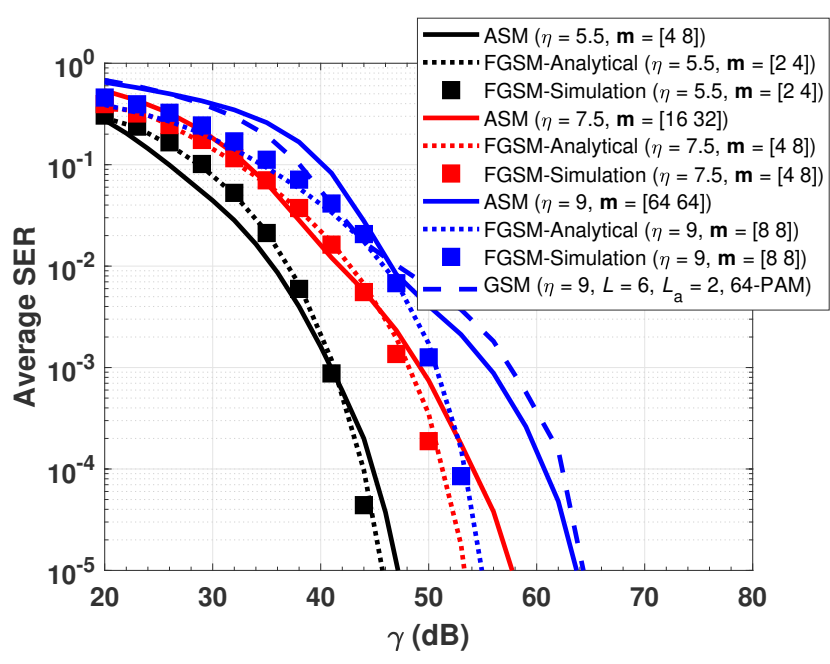

Fig. 2. Performance comparison of ASM and FGSM for $L=8$ and $U=2$ $\left(N_{1}=\frac{2^{\eta_{s}}}{2}, N_{2}=\frac{2^{\eta_{s}}}{2}\right)$.

$$
\operatorname{Add}_{\mathrm{ASM}}=(2 P-1) \sum_{u=1}^{U} N_{u} M(u) .
$$

It is worth noting that multiplications are more decisive in the decoding complexity compared with the additions. Since (15) and (17) are the same, the proposed FGSM provides almost the same decoding complexity as the ASM system.

\section{NUMERICAL RESUlTS AND Discussions}

In this section, we present Monte Carlo simulation results to confirm the accuracy of our SER derivation and provide performance comparisons with existing schemes to demons. We use an $8 \times 8$ VLC channel which is chosen from subsets of the general $16 \times 16 \mathrm{MIMO}$ system developed in [16]. We consider a fixed electrical transmit power for both ASM and proposed FGSM schemes in order to ensure a fair comparison. The channel state information is considered to be perfectly known at the receiver. The normalized channel matrix ${ }^{1}$ for the $8 \times 8$ MIMO setup is given by [17].

Figs. 2 and 3 present the average SER performance of the proposed FGSM compared to ASM for $L=8$ with fixed values of spectral efficiency. Moreover, a comparison with conventional GSM is also included. In Fig. 2, two groups (i.e., $U=2)$ are used $\left(N_{1}=2^{\eta_{s}} / 2, N_{2}=2^{\eta_{s}} / 2\right)$, whereas in Fig. 3 , three groups (i.e., $U=3)$ are utilized $\left(N_{1}=(3 / 8) 2^{\eta_{s}}\right.$, $\left.N_{2}=(1 / 4) 2^{\eta_{s}}, N_{3}=(3 / 8) 2^{\eta_{s}}\right)$. The modulation sizes for both ASM and FGSM are optimized according to the optimization formula given in (13).

It is observed that the derived closed-form expression of the proposed system given in (11) provides a close match with the simulated SER results. It is further observed from Figs. 2 and 3 that the proposed FGSM obtains better average SER results compared to ASM at the same spectral efficiency. For example, at the SER of $10^{-5}$ in Fig. 2, the required SNR

\footnotetext{
${ }^{1}$ It is assumed that $\mathbb{E}\left[\left\|\mathbf{h}_{l}\right\|^{2}\right]$ is a constant for $\forall l=1, \ldots, L$, i.e., the power for all columns of the MIMO VLC channel is constant [12, Ch. 3], where $\mathbf{h}_{l}$ is the $l$-th column of $\mathbf{H}$ and $\mathbb{E}[\cdot]$ refers to the expectation operator.
}

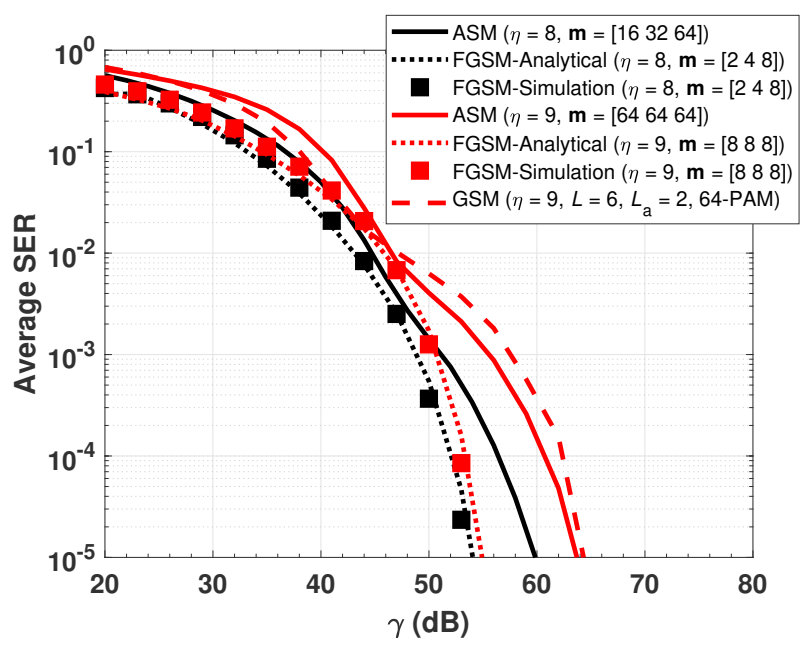

Fig. 3. Performance comparison of ASM and FGSM for $L=8$ and $U=3$ $\left(N_{1}=\frac{3}{8} 2^{\eta_{s}}, N_{2}=\frac{1}{4} 2^{\eta_{s}}, N_{3}=\frac{3}{8} 2^{\eta_{s}}\right)$.

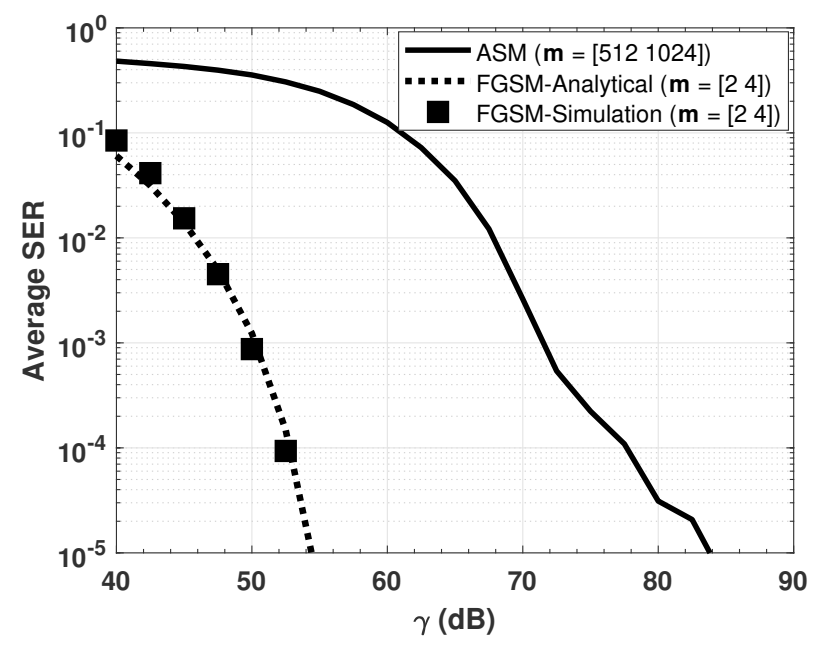

Fig. 4. Performance comparison of ASM and FGSM for $L=16, \eta=13.5$ bpcu and $U=2\left(N_{1}=\frac{2^{\eta_{s}}}{2}, N_{2}=\frac{2^{\eta_{s}}}{2}\right)$.

values are $47.2 \mathrm{~dB}$ and $57.7 \mathrm{~dB}$ for $\mathrm{ASM}$ to provide overall spectral efficiencies of $5.5 \mathrm{bpcu}$ and $7.5 \mathrm{bpcu}$, respectively. Whereas, the required SNR values to provide $5.5 \mathrm{bpcu}$ and $7.5 \mathrm{bpcu}$ are decreased to $45.7 \mathrm{~dB}$ and $53.3 \mathrm{~dB}$, respectively, by using the proposed FGSM system. This indicates that the FGSM improves the average SER by $1.5 \mathrm{~dB}$ and $4.4 \mathrm{~dB}$ for spectral efficiencies of $5.5 \mathrm{bpcu}$ and $7.5 \mathrm{bpcu}$, respectively. In Fig. 3, to achieve a spectral efficiency of $8 \mathrm{bpcu}$ at the SER of $10^{-5}$, the required SNR is $59.9 \mathrm{~dB}$ for ASM while this value decreases to $54.1 \mathrm{~dB}$ by using the proposed FGSM. This shows that the FGSM can improve the average SER performance by $5.8 \mathrm{~dB}$ for a spectral efficiency of $8 \mathrm{bpcu}$ in comparison with ASM. Moreover, it is observed that, at the same spectral efficiency, the proposed FGSM achieves a better SER in comparison with both ASM and conventional GSM in Figs. 2 and 3. For instance, at a spectral efficiency of $9 \mathrm{bpcu}$, the proposed FGSM scheme enhances the average SER by 9.5 $\mathrm{dB}$ in comparison to both ASM and conventional GSM. 


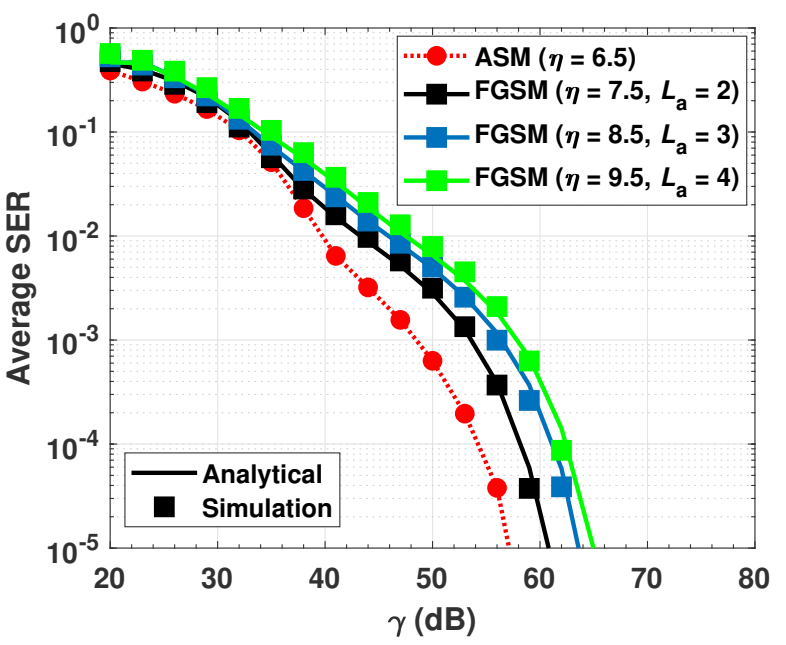

Fig. 5. Performance comparison of ASM and FGSM for $\mathbf{m}=\left[\begin{array}{ll}4 & 32\end{array}\right], L=8$ and $U=2\left(N_{1}=\frac{2^{\eta_{s}}}{2}, N_{2}=\frac{2^{\eta_{s}}}{2}\right)$.

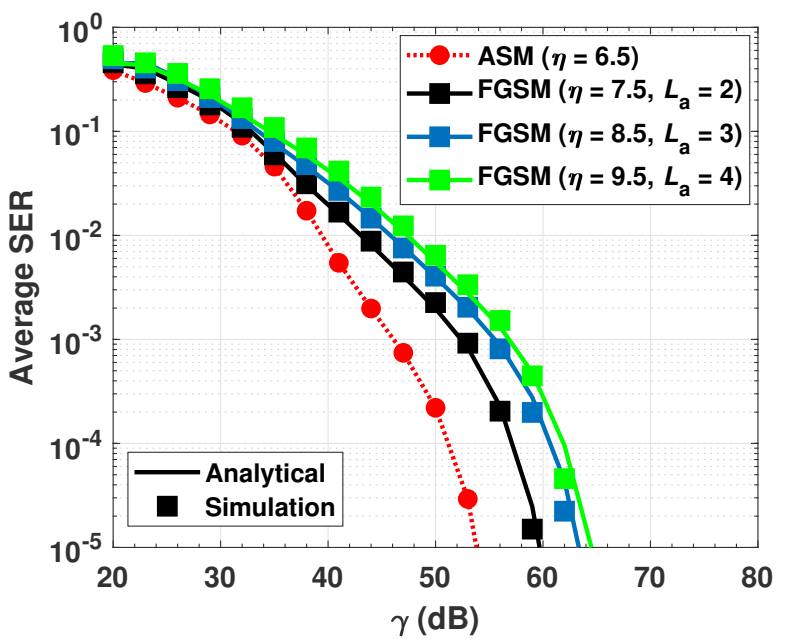

Fig. 6. Performance comparison of ASM and FGSM for $\mathbf{m}=\left[\begin{array}{lll}4 & 8 & 32\end{array}\right]$, $L=8$ and $U=3\left(N_{1}=\frac{1}{4} 2^{\eta_{s}}, N_{2}=\frac{3}{8} 2^{\eta_{s}}, N_{3}=\frac{3}{8} 2^{\eta_{s}}\right)$.

In Fig. 4, the average SER performance comparison of the FGSM and ASM schemes is presented for $L=16, \eta=13.5$ bpcu and $U=2\left(N_{1}=2^{\eta_{s}} / 2, N_{2}=2^{\eta_{s}} / 2\right)$. The $16 \times 16$ MIMO VLC channel given in [16] is normalized and used in Fig. 4. The SER performances for both ASM and FGSM are optimized using (13) in order to choose the optimal modulation size. It can be seen from Fig. 4 that the proposed FGSM significantly outperforms the ASM scheme at the same spectral efficiency. In a comparison with Fig. 2 presented for $L=8$, it is obvious that the use of more number of LEDs leads to a significant SER improvement.

In Figs. 5 and 6, we provide a SER comparison for FGSM and ASM schemes with the same modulation sizes and $L=$ 8. Fig. 5 represents the performance comparison for $U=2$ $\left(N_{1}=2^{\eta_{s}} / 2, N_{2}=2^{\eta_{s}} / 2\right)$ and $\mathbf{m}=[432]$. Fig. 6 shows the performance comparison for $U=3\left(N_{1}=(3 / 8) 2^{\eta_{s}}, N_{2}=\right.$ $\left.(1 / 4) 2^{\eta_{s}}, N_{3}=(3 / 8) 2^{\eta_{s}}\right)$ and $\mathbf{m}=\left[\begin{array}{lll}4 & 8 & 32\end{array}\right]$. As shown in
Figs. 5 and 6, while ASM can only provide an overall spectral efficiency of $6.5 \mathrm{bpcu}$, the proposed FGSM can improve the overall spectral efficiency by varying the number of active LEDs $\left(L_{a}\right)$. For instance, the FGSM obtains overall spectral efficiency values of 7.5, 8.5 and 9.5 bpcu for $L_{a}=2,3$ and 4, respectively, which results in relative improvements of $15.4 \%$, $30.8 \%$ and $46.2 \%$, respectively.

\section{CONCLUSiON}

In this paper, a novel FGSM system has been proposed in order to achieve better average SER and higher spectral efficiency compared to ASM with a fixed total number of LEDs. For the proposed FGSM system, the closed-form expression of the approximate average SER has been derived and confirmed by Monte-Carlo simulation, as well as, its decoding complexity has been analyzed. For example, at almost no extra cost in decoding complexity, the proposed system provides achievement up to $46.2 \%$ increase in spectral efficiency for a system with 8 LEDs.

\section{REFERENCES}

[1] Z. Ghassemlooy, L. N. Alves, S. Zvanovec, and M.-A. Khalighi, Visible Light Communications: Theory and Applications. CRC Press, 2017.

[2] Z. Wang, Q. Wang, W. Huang, and Z. Xu, Visible Light Communications: Modulation and Signal Processing. Hoboken, NJ, USA: Wiley, 2017.

[3] T. Fath and H. Haas, "Performance comparison of MIMO techniques for optical wireless communications in indoor environments," IEEE Trans. Commun., vol. 61, no. 2, pp. 733-742, Feb. 2013.

[4] S. Dimitrov and H. Haas, Principles of LED Light Communications: Towards Networked Li-Fi. Cambridge, U.K.: Cambridge University Press, Mar. 2015.

[5] X. Gao, Z. Bai, P. Gong, and D. O. Wu, "Design and performance analysis of LED-grouping based spatial modulation in the visible light communication system," IEEE Trans. Veh. Technol., vol. 69, no. 7, pp. 7317-7324, July 2020.

[6] J. Wang, H. Ge, M. Lin, J. Wang, J. Dai, and M. Alouini, "On the secrecy rate of spatial modulation-based indoor visible light communications," IEEE J. Sel. Areas Commun., vol. 37, no. 9, pp. 2087-2101, Sep. 2019.

[7] K. Xu, H. Yu, and Y. Zhu, "Channel-adapted spatial modulation for massive MIMO visible light communications," IEEE Photon. Technol. Lett., vol. 28, no. 23, pp. 2693-2696, Dec. 2016.

[8] S. P. Alaka, T. Lakshmi Narasimhan, and A. Chockalingam, "Generalized spatial modulation in indoor wireless visible light communication," 2015 IEEE Global Communications Conference (GLOBECOM), San Diego, CA, 2015, pp. 1-7.

[9] C. R. Kumar and R. K. Jeyachitra, "Dual-mode generalized spatial modulation MIMO for visible light communications," IEEE Commun. Lett., vol. 22, no. 2, pp. 280-283, Feb. 2018.

[10] J.-Y. Wang , J.-X. Zhu, S.-H. Lin, and J.-B. Wang "Adaptive spatial modulation based visible light communications: SER analysis and optimization," IEEE Photon. J., vol. 10, no. 3, pp. 1-14, June 2018.

[11] J. G. Proakis and M. Salehi, Digital Communications. New York, USA: McGrawHill, Inc., 5th ed., 2008.

[12] R. Mesleh and A. Alhassi, Space Modulation Techniques. Hoboken, NJ, USA: Wiley, 2018.

[13] Z. B. Zabinsky, Stochastic Adaptive Search for Global Optimization. Kluwer Academic Publishers, 2003.

[14] G. H. Givens and J. A. Hoeting, Computational Statistics. Hoboken, NJ, USA: Wiley, 2nd ed., 2013.

[15] L. Bai and J. Choi, Low Complexity MIMO Detection. Springer, Boston, MA, USA, 2012.

[16] O. Narmanlioglu, R. C. Kizilirmak, T. Baykas, and M. Uysal, "Link adaptation for MIMO OFDM visible light communication systems," IEEE Access, vol. 5, pp. 26006-26014, 2017.

[17] M. Al-Nahhal, E. Basar, and M. Uysal, "Adaptive unipolar MIMOOFDM for visible light communications," 2019 European Conference on Networks and Communications (EuCNC), Valencia, Spain, 2019, pp. 73-77. 